\title{
MAGIC detection of sub-TEV emission from gravitationally lensed blazar QSO B0218+357
}

\author{
D. Dominis Prester ${ }^{1}$, J. Sitarek ${ }^{2}$, J. Becerra ${ }^{3,4}$, S. Buson ${ }^{5,4}$, \\ E. Lindfors ${ }^{6}$, M. Manganaro ${ }^{7}$, D. Mazin ${ }^{8}$, M. Nievas Rosillo ${ }^{9}$, \\ K. Nilsson ${ }^{6}$, A. Stamerra ${ }^{10}$, F. Tavecchio ${ }^{11}$, Ie. Vovk $^{12}$ for \\ the MAGIC Coll. ${ }^{13}$ and the Fermi-LAT Coll. ${ }^{14}$
}

\author{
${ }^{1}$ University of Rijeka, Department of Physics, 51000 Rijeka, Croatia, email: \\ dijana@phy.uniri.hr; ${ }^{2}$ University of Łódź, PL-90236 Lodz, Poland; ${ }^{3}$ NASA Goddard Space \\ Flight Center, Greenbelt, MD 20771, USA; ${ }^{4}$ Department of Physics and Department of \\ Astronomy, University of Maryland, MD 20742, USA; ${ }^{5}$ Università di Padova and INFN, \\ I-35131 Padova, Italy; ${ }^{6}$ Tuorla Observatory, Department of Physics and Astronomy, University \\ of Turku, Finland; ${ }^{7}$ IAC, E-38200 La Laguna; Universidad de La Laguna, Dpto. Astrofísica, \\ E-38206 La Laguna, Tenerife, Spain; ${ }^{8}$ ICRR, The University of Tokyo, 277-8582 Chiba, Japan; \\ ${ }^{9}$ Universidad Complutense, E-28040 Madrid, Spain; ${ }^{10}$ INAF Osservatorio Astrofisico di Torino, \\ I-10025 Pino Torinese, Italy; ${ }^{11}$ INAF National Institute for Astrophysics, I-00136 Rome, Italy; \\ ${ }^{12}$ Max-Planck-Institut für Physik, D-80805 München, Germany; \\ ${ }^{13}$ http://wwwmagic.mppmu.mpg.de; ${ }^{14}$ http://fermi.gsfc.nasa.gov/
}

Keywords. Gamma rays: galaxies - Gravitational lensing - Galaxies: jets - Quasars: individual: QSO B0218+357

The blazar QSO B0218+357 is the first gravitationally lensed blazar detected in the very high energy (VHE, $E>100 \mathrm{GeV}$ ) gamma-ray spectral range (Ahnen et al. 2016). It is gravitationally lensed by the intervening galaxy B0218+357G $\left(z_{l}=0.68466 \pm 0.00004\right.$, Carilli et al.1993), which splits the blazar emission into two components, spatially indistinguishable by gamma-ray instruments, but separated by a 10-12 days delay. In July 2014 a flare from QSO B0218+357 was observed by the Fermi-LAT (Large Area Telescope, Atwood et al. 2009, Ackermann et al. 2012), and followed-up by the MAGIC (Major Atmospheric Gamma Imaging Cherenkov) telescopes, a stereoscopic system of two $17 \mathrm{~m}$ Imaging Atmospheric Cherenkov Telescopes located on La Palma, Canary Islands (Aleksić et al. 2016a, 2016b), during the expected time of arrival of the delayed component of the emission. MAGIC could not observe the leading image due to the Full Moon. The MAGIC and Fermi-LAT observations were accompanied by optical data from KVA telescope at La Palma, and X-ray observations by Swift-XRT (Fig. 1 left). Variability in gamma-rays was of the order of one day, while no variability correlated with gamma-rays was observed at lower energies. The flux ratio of the leading to trailing image in HE gamma-rays was larger than in the flare of QSO B0218+357 observed by Fermi-LAT in 2012 (Cheung et al. 2014). Changes in the observed flux ratio can be caused by gravitational microlensing on individual stars in the host galaxy (Neronov et al. 2015), or by other compact objects like for ex. clumps in giant molecular clouds (Sitarek \& Bednarek 2016).

We derive gamma-ray spectral energy distribution (Fig. 1, right top) and model it in multiwavelength context (Fig. 1, right bottom). Large separation of the two peaks and strong Compton dominance, together with the MWL light curve features described above, lead us to the conclusion that a two zone external Compton model can be suitable for describing this source. According to this model, gamma-rays $(\mathrm{GeV}$ and sub- $\mathrm{TeV})$ are produced beyond the Broad Line Region (BLR) as a sum od the SSC and EC components on the radiation field from BLR and dusty torus (Jet out), while optical emission and X-rays are produced within the BLR (Jet in).

QSO B0218+357 is one of the two most distant AGN ( $\mathrm{z}=0.944)$ detected so far in the VHE gamma-ray range, together with PKS $1441+25(\mathrm{z}=0.940)$ which was also detected by 

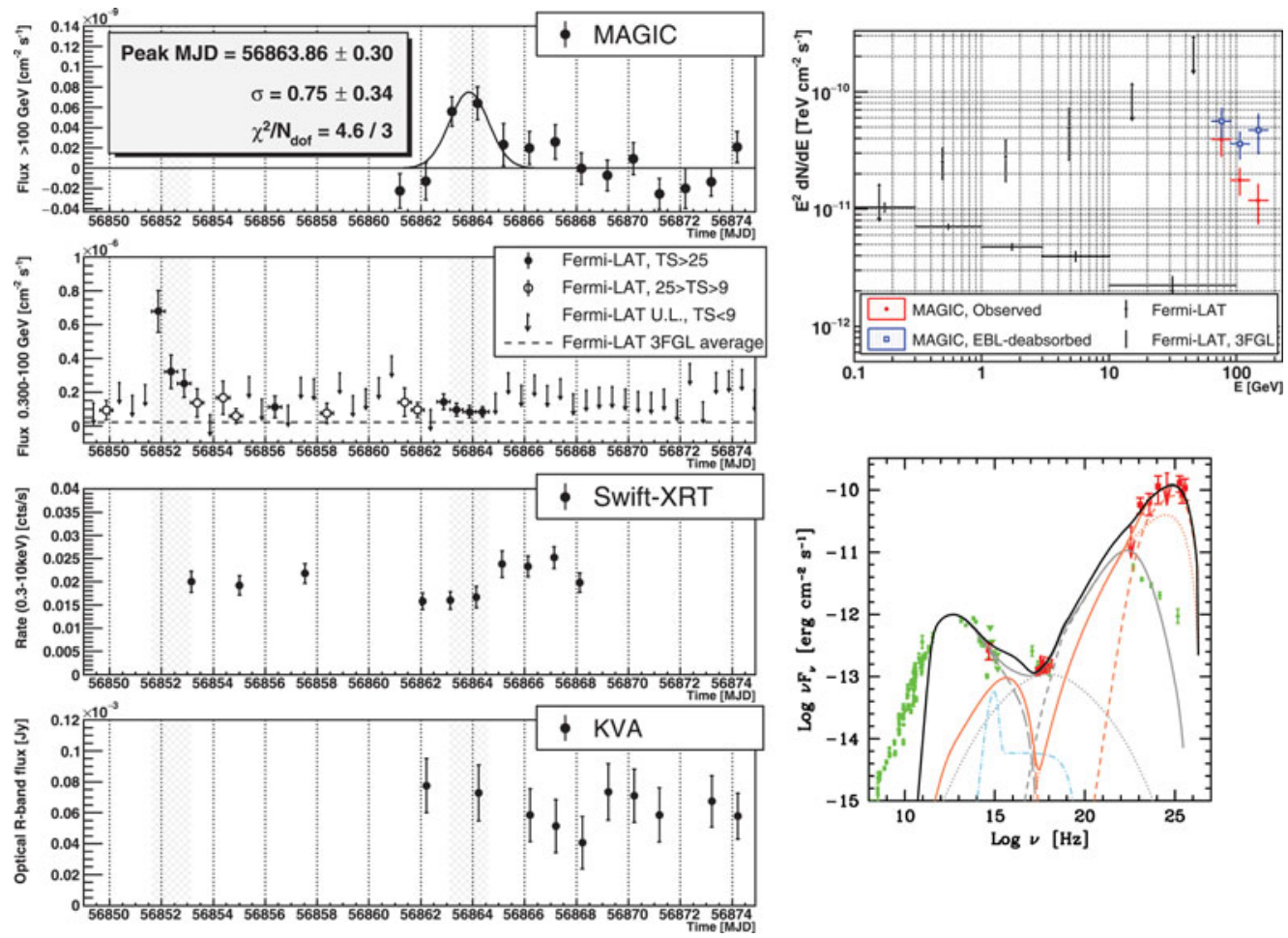

Figure 1. July 2014 QSO B0218+357 flare (Ahnen et al. 2016). Left: MWL light curve. The two shaded regions are separated by 11.46 days. Right top: Gamma-ray SED. MAGIC observations during MJD $=56863-56864$ before and after deabsorption due to EBL compared to the Fermi-LAT spectrum from the same time and the average emission of QSO B0218+357 in the 3FGL catalog. Right bottom: Broadband SED. The reconstructed fluxes (squares) are corrected for different magnifications in different energy ranges. Filled circles: historical measurements (flux upper limit). Emission from the region located within (left thin solid line) and beyond (right thin solid line) the BLR. Long dashed curves: synchrotron radiation; Dotted: SSC emission; Short dashed: the external Compton emission. Dash-dot line: accretion disk emission and its X-ray corona. Thick black solid line: the sum of the non-thermal emission from both regions.

the MAGIC telescopes (Ahnen et al. 2015). The GeV and sub-TeV data, obtained by FermiLAT and MAGIC, are used to set some constraints on the extragalactic background light (EBL) that are compatible with current models.

\section{References}

Ackermann, M., et al. 2012, ApJS, 203, 4

Ahnen, M. L., et al. 2015, ApJL, 815, 2, L23

Ahnen, M. L., et al. 2016, http://adsabs.harvard.edu/abs/2016arXiv160901095M

Aleksić, J., et al. 2016a, Astroparticle Physics, 72, 61

Aleksić, J., et al. 2016b, Astroparticle Physics, 72, 76

Atwood W. B., et al. 2009, ApJ, 697, 1071

Cheung, C. C., Larsson, S., Scargle, J. D., et al. 2014, ApJL, 782, L14

Carilli, C. L., Rupen, M. P., \& Yanny, B. 1993, ApJL, 412, L59

Domínguez, A., Primack, J. R., Rosario, D. J., et al. 2011, MNRAS, 410, 2556

Neronov, A., Vovk, I., \& Malyshev, D. 2015, Nature Physics, 11, 664

Sitarek, J. \& Bednarek, W. 2016, MNRAS, 459, 1959 